\title{
Custo de implantação e planejamento de uma piscicultura de grande porte no Estado de Mato Grosso, Brasil
}

Barros, A.F. ${ }^{\circledR} ;$ Maeda, M.M. ${ }^{2}$; Maeda, A. ${ }^{2}$; Silva, A.C.C. ${ }^{1}$ e Angeli, A.J. ${ }^{2}$

\begin{abstract}
Universidade do Estado de Mato Grosso. UNEMAT. Departamento de Zootecnia. Campus Universitário de Pontes e Lacerda. MT. Brasil. ${ }^{2}$ Instituto de Educação. Consultoria e Assessoria Universitária. ABRACE BRASIL. Faculdades Integradas do Vale do lguaçu. UNIGUAÇU. União da Vitória. PR. Brasil.
\end{abstract}

\section{PALAVRAS CHAVE ADICIONAIS}

Indicadores de rentabilidade.

Peixes redondo.

Pintado da Amazônia.

Tanques escavados.

\section{RESUMO}

Objetivou-se com este trabalho analisar a viabilidade econômica da implantação de uma piscicultura de grande porte no Estado de Mato Grosso, com 174 ha de lâmina d'água em sistema de tanques escavados, utilizando $30 \%$ dessa área com criação do pintado da Amazônia Pseudoplatystoma spp. e 70\% com criação de peixes redondo (espécies e híbridos do gênero Colossoma e Piaractus). Os dados foram coletados diretamente no setor produtivo, em que estão sendo construídos 73 tanques escavados, com sistemas de abastecimento e drenagem individual. Foram determinados ciclo de criação de 12 meses e os seguintes indicadores zootécnicos, para os peixes redondo e pintado, respectivamente: biomassa de estocagem final média de $0,799 \mathrm{~kg} / \mathrm{m}^{-2}$ e de $0,802 \mathrm{~kg} / \mathrm{m}^{-2}$, conversão alimentar aparente de 1,80 e 1,90, taxa de sobrevivência de $75 \%$ e de $86 \%$, peso médio final dos peixes de $1,8 \mathrm{~kg}$ e de $2,0 \mathrm{~kg}$. Para obtenção do custo total médio de produção do quilograma dos peixes, foi utilizada a metodologia do custo total de produção (CTP). Para análise de rentabilidade foram utilizados os seguintes indicadores: receita bruta (RB), lucro (L), margem de contribuição (MC), valor presente líquido (VPL), período de retorno do capital (PRC) e taxa interna de retorno (TIR). Para instalação da piscicultura serão investidos $R \$ 8.688 .933,24$. O custo total médio do quilograma do peixe, produção total e preço de comercialização dos peixes redondo e do pintado da Amazônia serão, respectivamente: $R \$ 3,51$ e $R \$ 5,14$; $972.797 \mathrm{~kg}$ e $418.639 \mathrm{~kg}, R \$ 4,00$ e $R \$ 7,00$, o qual totalizará lucro de $R \$ 1.251 .628,29$ ao ano (US\$1=R\$2,23 cotação média em Julho de 2014). Os resultados do VPL, PRC e TIR para o horizonte de 15 anos são, respectivamente, $R \$ 7.239 .606,49 ; 4,03$ anos e $23,4 \%$. Foi realizada análise de sensibilidade com diversos cenários desfavoráveis, ainda assim todos apresentaram viabilidade econômica.

\section{Cost of the implementation and planning of large size fish farming in the State of} Mato Grosso, Brazil

\section{SUMMARY}

The aim of this study was to analyze the economic feasibility of implementing a large fish farm in Mato Gross, Brazil, with 174 ha of water surface under dug fishpond systems, using a $30 \%$ of this area for the farming of Amazonian catfish Pseudoplatystoma spp. and a $70 \%$ for the farming of round fish (Colossoma, Piaractus genus and their hybrids). The data were directly collected from the productive sector, which 73 fishponds were being built in, with individual supply and drainage systems. The creation of a 12 months cycle and the following zootechnical indicators for round fish and Amazonian catfish were consecutively determined: $0.799 \mathrm{~kg} / \mathrm{m}^{-2}$ and $0.802 \mathrm{~kg} / \mathrm{m}^{-2}$ average final biomass storage, 1.80 and 1.90 apparent feed conversion, $75 \%$ and $86 \%$ survival rate, and $1.8 \mathrm{~kg}$ to $2.0 \mathrm{~kg}$ fish final weight. The Total Production Cost methodology (TCC) was used in order to obtain the total average cost for each fish kilogram produced. The following indicators were used for its profitability analysis: gross Income (GI), profit (P), contribution margin (CM), net present value (NPV), capital return period (CRP) and internal rate of return (IRR). $R \$ 8,688,933.24$ will be invested for fish farming installation. The average total cost, total production and the commercializing price of round fish and Amazonian catfish will respectively be, $R \$ 3,51$ and $R \$ 5,14,972,797 \mathrm{~kg}$ and $418,639 \mathrm{~kg}, R \$ 4,00$ and $R \$ 7,00$, which will result in a total income of $R \$ 1.251 .628,29$ per year (US\$ $1=R \$ 2.23$ average price in July 2014). The results of the NPV, IRR, CRP 15 years ahead are consecutively R $\$ 7.239 .606,49 ; 4,03$ years and $23,4 \%$. Sensitivity analysis was performed with several adverse scenaries and all of them showed economic viability.

isso contribui com desenvolvimento de outras atividades relacionadas, como indústrias de rações, transporte dos peixes, equipamentos tecnológicos, entre outras (Barros e Martins, 2012). Seu potencial de crescimento
A piscicultura no estado de Mato Grosso está em constante crescimento dentro do setor agropecuário e 
no Estado ainda é maior, devido sua abundância recursos hídricos, elevada extensão territorial, clima tropical e significativa produção de grãos que são utilizados na fabricação de ração, mercado consumidor e criação de espécies nativas adaptadas ao sistema de criação.

Apesar de constituir um sistema de produção agropecuária, para conseguir os lucros esperados, faz-se necessária utilização de métodos modernos e adequados com base em conceitos ecológicos, científicos, tecnológicos e econômicos (Sabaag et al., 2007). Desse modo, no planejamento de uma piscicultura são importantes os aspectos econômicos da atividade, pois proporciona a escolha entre cenários de alternativas de investimentos em relação ao tempo de retorno, rentabilidade e lucratividade.

Esses resultados econômicos também serão influenciados diretamente pelos indicadores zootécnicos da piscicultura. $\mathrm{O}$ que torna seu controle essencial para que se tenha sucesso na produção com menor custo de produção. A alimentação, por exemplo, em momentos pré-definidos contribui para a redução da conversão alimentar, incremento do ganho de peso, entre outros. E o conhecimento da quantidade correta de arraçoamento possibilita a diminuição do desperdício do alimento reduzindo os custos (Dieterich et al., 2013).

No entanto, ainda há carência de informações técnicas e científicas, relacionadas a indicadores econômicos e zootécnicos na área de piscicultura, principalmente relacionadas a empreendimentos de grande porte e com criação de espécies nativas. Diante desses fatores, objetivou-se com este trabalho determinar o investimento da implantação e os indicadores zootécnicos e econômicos de uma piscicultura de grande porte, com 174 ha de lâmina d'água, que está em fase de implantação no estado de Mato Grosso. O que possibilitará o produtor em melhores tomadas de decisões econômicas e tecnológicas, o que o torna mais capacitado para as oscilações do mercado.

\section{MATERIAL E MÉTODOS}

A piscicultura está em fase de implantação, localizada no do Estado de Mato Grosso, Brasil. Nesse estado, as pisciculturas são classificadas por área de lâmina d'água de acordo com a Lei Estadual nº 8.464 de 04.04.2006 Art. $3^{\circ}$, em: Micro - até um hectare; Pequena - entre 1,1 e 5 hectares; Média - entre 5,1 e 50 hectares e Grande - maior que 50 hectares (Diário Oficial, 2006).

Nessa classificação a piscicultura estudada foi caracterizada como de grande porte, com área total de lâmina d'água de 174 ha divididos em 45 tanques escavados com média de 3 ha por tanque e 28 tanques escavados com média 1,4 ha de lâmina d'água (figura 1), com sistemas de abastecimento e drenagem individual. $\mathrm{Na}$ elaboração do projeto foram computados investimentos com aquisição da área, infraestrutura, aquisição de máquinas e equipamentos, taxas com licenciamento ambiental e elaboração do projeto.

Da área total de lâmina d'água, 121,8 ha (70\%) serão disponibilizados para criação do grupo dos peixes redondo (espécies e híbridos do gênero Colossoma e Piaractus) e 52,2 ha (30\%) para a criação do pintado

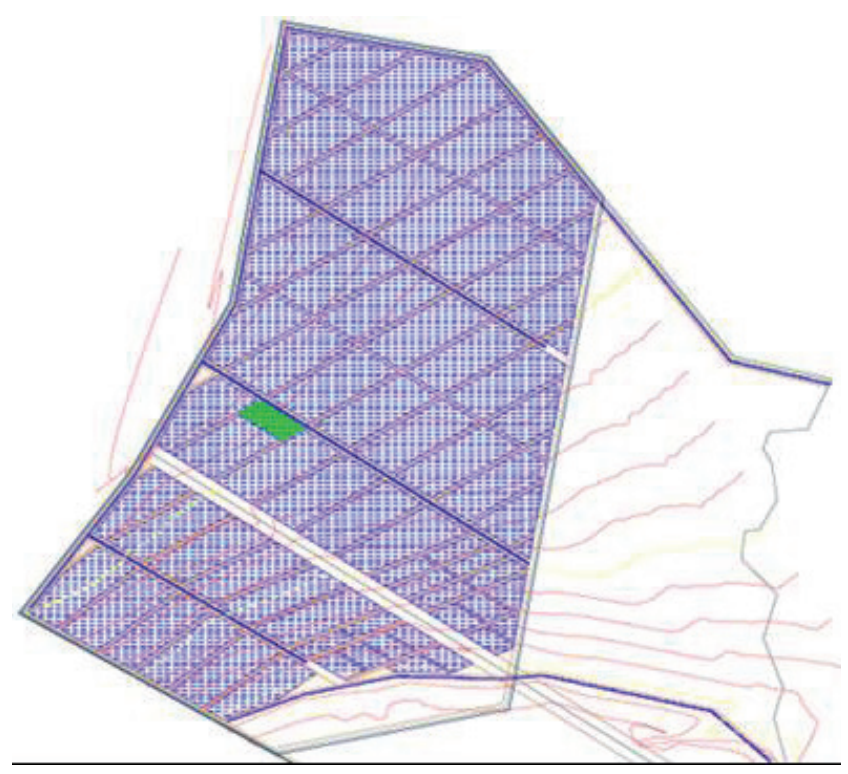

Figura 1. Planta baixa da distribuição dos tanques escavados da piscicultura com 174 ha de lâmina d'água em fase de instalação, Estado de Mato Grosso, Brasil (Floor plan of the distribution of ponds, fish farm with a 174 ha of water surface during the installation phase, State of Mato Grosso, Brazil).

da Amazônia, híbrido resultante do cruzamento entre o cachara (Pseudoplatystoma punctifer) e o jundiá (Leiarius marmoratus).

O sistema de criação dos peixes será dividido em duas fases, I e II. Os juvenis dos peixes redondos serão adquiridos com 3-5 cm de comprimento e peso médio de 5 g e os juvenis de pintado da Amazônia com 6-8 cm de comprimento com peso médio de $10 \mathrm{~g}$. A alimentação de ambas as espécies será com ração comercial extrusada dividida em 3 a 4 tratos na fase I e na II de 1 a 2 tratos diários. A ração comercial utilizada para os juvenis de peixes redondo na fase I será com $40 \%$ de proteína bruta (PB), e na II com $36 \%$, 32\% e $28 \%$ de PB para terminação dos mesmos. Para os juvenis de pintado da Amazônia a ração extrusada utilizada será para peixes carnívoros, na fase I com $45 \%$ de PB, e na fase II $40 \%$ e $36 \%$ de PB.

Os indicadores zootécnicos estabelecidos foram baseados na média de resultados obtidos diretamente no setor produtivo. A conversão alimentar aparente (CAA) foi calculada a partir da quantidade de ração consumida dividida pelo ganho de peso alcançado no final de cada fase do ciclo de criação. A taxa de sobrevivência foi obtida por meio do número de animais despescados dividido pelo número de animais estocados multiplicado por 100, expressos em porcentagem. A biomassa de estocagem é a quantidade em quilograma de peso vivo por metro quadrado de lâmina d'água $\left(\mathrm{kg} \mathrm{m}^{-2}\right)$. O peso médio final foi calculado pelo peso total despescado dividido pelo número de peixes, expresso em $\mathrm{kg}$.

Na determinação do custo total de produção (CTP) foi utilizada a metodologia descrita por Martins e Borba (2008). Foram consideradas para a estrutura de custo de produção, além dos desembolsos que ocorrerão no processo produtivo, às depreciações dos itens de 
capital fixo, bem como as remunerações ou custo de oportunidades dos fatores de produção empregados (Scorvo Filho et al., 2004). Em que, o CTP corresponde ao somatório do custo fixo (CF) e custo variável (CV).

Foi realizado levantamento de preços de mercados praticados no Estado de Mato Grosso, no mês de julho de 2014, relacionados aos itens de investimentos em benfeitorias, máquinas, equipamentos e despesas operacionais necessários para o sistema de criação dos peixes.

Adotou-se ainda para o cálculo do custo total de produção os seguintes métodos:

- Para remuneração dos itens de investimento, o método selecionado foi o de aplicação financeira de baixo risco, rendimento da caderneta de poupança com juros de $6,75 \%$ a.a. sobre o valor do capital fixo médio investido;

- Para remuneração do capital circulante o método adotado foi o de crédito de Custeio Rural do Banco do Brasil de 7,95\% a.a.;

- Pelo método linear, considerando o valor de sucata igual a zero, determinou-se depreciação das benfeitorias, máquinas e equipamentos;

- Reparos e manutenção dos equipamentos foram determinados a taxa de $5 \%$ ao ano sobre o valor de aquisição do bem; para benfeitorias, essa taxa foi de $2 \%$ ao ano do valor das construções (Martin et al., 1995);

- Ainda foi considerada taxa de 5\% sobre o valor das despesas efetivas, para eventualidades, com finalidade de cobrir alguma situação imprevista ou falha de informação;
- A mão de obra contratada teve como remuneração dois salários mínimos, considerando a função desempenhada, mais os encargos sociais, como décimo terceiro, férias, Instituto Nacional de Seguridade Social (INSS) entre outros, que correspondem a 42,9\% do salário;

- Foram levados em consideração os valores computados para o cadastramento ambiental rural, outorga de direito de uso dos recursos hídricos, licença prévia e licença de instalação da piscicultura;

- Para elaboração do projeto com topografia e planta baixa foi calculado com base em $1,4 \%$ do total do investimento.

Para análise de rentabilidade, com horizonte do projeto para 15 anos foram empregados os seguintes indicadores:

- Receita bruta (RB): obtida por meio da venda da produção, o valor encontrado pela multiplicação entre a quantidade comercializada pelo preço de venda;

- Lucro (L): RB-CTP;

- Margem de contribuição (MC): RB-CV;

- Custos médio (CVMe, CFMe, CTMe): são referentes aos custos por unidade de produção, representados pela relação existente entre os custos e a quantidade produzida, expresso em $\mathrm{R} \$ \mathrm{~kg}^{-1}$;

- Valor presente líquido (VPL): compara todas as receitas e saídas de capital na data inicial do investimento, diminuindo todos os valores futuros do fluxo de caixa a taxa de juros estabelecida, avaliando o custo do capital;

Tabela I. Relação e valores dos itens de investimentos para instalação de uma piscicultura com 174 ha de lâmina d'água, Estado de Mato Grosso, Brasil, 2014 (Relation and values of investment items for the installation of a fish farm with 174 ha water surface, State of Mato Grosso, Brazil, 2014). (US\$1= R \$2,23: cotação média em Julho de 2014/average price in July 2014).

\begin{tabular}{|c|c|c|c|}
\hline Itens & Especificações & Qtdade & Valor Total $(\mathrm{R} \$)$ \\
\hline Depósito de ração $25 \mathrm{~m} \times 18 \mathrm{~m}\left(\mathrm{~m}^{2}\right)$ & Pré-moldado/Telha zincada & 450 & $126.000,00$ \\
\hline \multirow{3}{*}{$\begin{array}{l}\text { Construção } 174 \text { ha de lâmina d'água } \\
73 \text { tanques escavados (hora máquina) }\end{array}$} & Escavadeira PC & 500 & $110.000,00$ \\
\hline & Scrapers tratorizado & 12.180 & $2.801 .400,00$ \\
\hline & Trator de esteiras & 3.480 & $765.600,00$ \\
\hline Construção de canais (m) & $2390 \mathrm{~m} \times 4 \mathrm{~m} \times 1,5 \mathrm{~m}$ & 2.390 & $133.862,00$ \\
\hline Tubulações PAD $(\mathrm{m})$ & Diâmetro de 40 e 60 cm & 11.400 & $1.783 .800,00$ \\
\hline Casas de funcionários $\left(93 \mathrm{~m}^{2}\right)$ & \multirow{3}{*}{ Alvenaria/Forrada/Telha de barro } & 3 & $360.000,00$ \\
\hline Casa sede $\left(150 \mathrm{~m}^{2}\right)$ & & 1 & $180.000,00$ \\
\hline Alojamento - Funcionários $\left(60 \mathrm{~m}^{2}\right)$ & & 1 & $80.000,00$ \\
\hline Poço artesiano & Profundidade de $100 \mathrm{~m}$ & 1 & $25.000,00$ \\
\hline Instalação elétrica & Complexo sede geral & 1 & $80.000,00$ \\
\hline Auto propelido/Tratador ração & Trator acoplado/2009 & 1 & $110.000,00$ \\
\hline Trator MF 275 (usado) & MF 275/2007 & 1 & $30.000,00$ \\
\hline Automóvel Utilitário & S-10/Chevrolet/2014 & 1 & $80.000,00$ \\
\hline Redes de despesca & 180,60 e $30 \mathrm{~m}$ com 40, 10 e $6 \mathrm{~mm}$ & 3 & $17.200,00$ \\
\hline Barco e motor Yamaha $15 \mathrm{HP}$ & Alumínio - $5 \mathrm{~m}$ & 2 & $15.000,00$ \\
\hline Balança eletrônica & Toledo - 500 kg & 1 & $2.100,00$ \\
\hline Multiparâmetro & Bernauer & 1 & $16.000,00$ \\
\hline Outros* & & & $337.268,10$ \\
\hline Aquisição da área (ha) & Licenciamento e projeto & 368 & $1.635 .703,14$ \\
\hline TOTAL & & & $8.688 .933,24$ \\
\hline
\end{tabular}


Tabela II. Indicadores zootécnicos estimados para criação de peixes redondo em 121,8 ha e de pintado da Amazônia em 52,2 ha de lâmina d'água de tanques escavados, Estado de Mato Grosso, Brasil (Estimated zootechnical indicators for creation of round fish in 121.8 ha and Amazon pintado in 52,2 ha of water surface of fishpounds, State of Mato Grosso, Brazil).

\begin{tabular}{|c|c|c|c|c|c|}
\hline \multirow{2}{*}{ Parâmetros de desempenho } & \multirow{2}{*}{ Un. } & \multicolumn{2}{|c|}{ Peixes redondo } & \multicolumn{2}{|c|}{ Pintado da Amazônia } \\
\hline & & Fase I & Fase II & Fase I & Fase II \\
\hline Quantidade de peixes inicial & $\mathrm{n}^{\circ}$ & 718.674 & 574.939 & 244.818 & 220.336 \\
\hline Peso médio inicial & $\mathrm{kg}$ & 0,005 & 0,150 & 0,010 & 0,200 \\
\hline Quantidade de peixes final & $\mathrm{n}^{\circ}$ & 574.939 & 540.443 & 220.336 & 209.319 \\
\hline Peso médio final & $\mathrm{kg}$ & 0,150 & 1,800 & 0,200 & 2,000 \\
\hline Peso total despescado & $\mathrm{kg}$ & 86.241 & 972.797 & 44.067 & 418.639 \\
\hline Biomassa de estocagem final & $\mathrm{kg} / \mathrm{m}^{-2}$ & 0,479 & 0,799 & 0,441 & 0,802 \\
\hline Conversão alimentar aparente & $\mathrm{kg}$ & 1,300 & 1,810 & 1,400 & 1,920 \\
\hline Taxa de mortalidade & $\%$ & $20 \%$ & $6 \%$ & $10 \%$ & $5 \%$ \\
\hline Período de cultivo & Meses & 3 & 9 & 3 & 9 \\
\hline Quantidade de ração & $\mathrm{kg}$ & 107.442 & 1.604 .667 & 58.267 & 719.177 \\
\hline
\end{tabular}

- Período de retorno do capital (PRC): é o período de tempo em que se consegue recuperar o capital investido;

- Taxa interna de retorno (TIR): representa a taxa de desconto que iguala em um único momento os fluxos de entradas e saídas de caixa;

- Relação benefício-custo (RBC): é a relação entre o valor atual dos retornos esperados e o valor dos custos estimados.

\section{RESULTADOS E DISCUSSÃO}

O investimento com benfeitorias, máquinas e equipamentos necessário para a implantação de uma piscicultura com 174 ha de lâmina d'água de tanques escavados é de R\$ 8.688.933,24 (tabela I). Sendo que o investimento, necessário exclusivamente com construção de cada hectare lamina d'água são de $\mathrm{R} \$ 31.463,57$, equivalentes a hora/máquina e investimentos com sistema de captação, abastecimento e escoamento de água. Vilela et al. (2013), verificaram investimento maior, de $\mathrm{R} \$ 35.053,50$ na construção de 1 ha de lâmina d'água, no entanto, dividido em oito tanquesvisto que, quanto maior o número de tanques por hectare de lâmina d’água, maior a quantidade de horas /máquina utilizadas, o que eleva o custo de implantação.

As condições de solo e topografia de diferentes regiões também interferem diretamente nos sistemas de preparo e construção de tanques e, consequentemente, no custo de implantação da piscicultura, devido à quantidade de horas/máquina utilizadas (Furnaleto e Esperancini, 2009). Esses fatores devem ser levados em consideração, pois, juntamente com a hidráulica, são os itens que apresentam maior participação com $63,01 \%$ ( $\$$ \$ 5.474.662,00) dos demais valores dos itens de investimento (tabela I).

Barros e Martins (2012) obtiveram proporções de investimentos próximos com a construção de 106,8 ha de lamina d'água, com participação de 71,4\% do capital imobilizado, porém o investimento em valores $(\mathrm{R} \$)$ por hectare foi menor, uma vez que esses tanques foram construídos em sistemas de barragem e tem menor custo do que os tanques construídos em sistemas de derivação, devido a simplicidade da construção e, consequentemente, se tornam mais econômico. Por isso a importância em se buscar topografias mais adequadas com a finalidade de diminuir os investimentos com esse item.

A tabela II apresenta os indicadores zootécnicos referentes à criação de peixes redondos e do pintado da Amazônia. Melo et al. (2001) também utilizaram o ciclo de criação de 12 meses para tambaqui Colossoma macropomum, e obtiveram resultados próximos com relação a taxa de sobrevivência que foi de $74 \%$. No entanto, Izel e Melo (2004) verificaram resultados superiores, com 95,4\% de taxa de sobrevivência trabalhando em 15 ha de lâmina d'água. Esse melhor resultado pode ter ocorrido devido às pisciculturas de pequeno porte ser mais eficiente no controle dos predadores do que em pisciculturas de grande porte.

A conversão alimentar aparente média para os peixes redondos no presente trabalho foi de 1,80 e, valores semelhantes foram obtidos por Barros e Martins (2012) e com mesmo período de ciclo de criação, obtendo peso médio final de $1,873 \mathrm{~kg}$. Barros et al. (2010) ressaltaram que a obtenção de melhor ou pior CAA está relacionada principalmente com a quantidade e qualidade da ração ofertada, contudo outros fatores também influenciam como, qualidade da água, juvenis e biomassa de estocagem. Esse é um índice zootécnico de importância, pois apresenta influência direta na produtividade, peso médio final dos peixes e, consequentemente, no CTP.

Os indicadores zootécnicos para criação de pintado da Amazônia apresentados na tabela II, apresenta taxa de sobrevivência total de $86 \%$ e CAA média de 1,90 esses se encontram entre os valores obtidos por CruzCasallas et al. (2010), que trabalharam com o desempenho produtivo do Leiarius marmoratus sob diferentes densidades de estocagem. A utilização dos peixes híbridos, como o pintado da Amazônia no presente estudo, é devida os mesmos apresentarem melhor desempenho que seus progenitores, porém ainda existe a necessidade do desenvolvimento de um programa de melhoramento genético que faça a seleção da espécie pura para o acréscimo da produção comercial (Oliveira et al., 2014). 
Tabela III. Custo total de produção de $972.797 \mathrm{~kg}$ de peixes redondo e $418.639 \mathrm{~kg}$ de pintado da Amazônia em 174 ha de lâmina d'água, Estado de Mato Grosso, Brasil (Total production cost of $972.797 \mathrm{~kg}$ of round fish and $418.639 \mathrm{~kg}$ of Amazon pintado on 174 ha of water surface, State of Mato Grosso, Brazil). (US\$ 1= R\$ 2,23: cotação média em Julho de 2014/average price in July 2014).

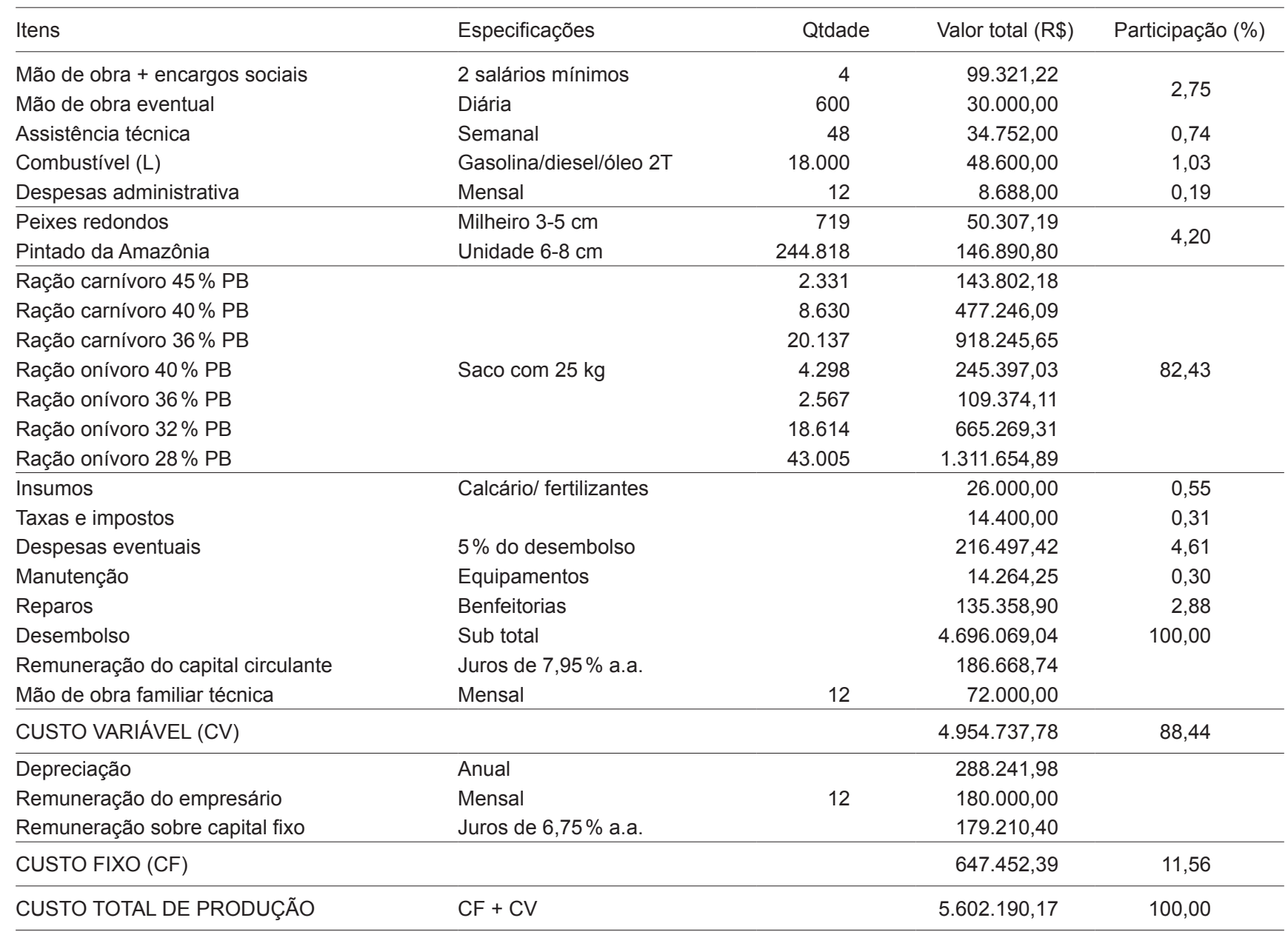

A tabela III se refere ao custo total de produção (CTP) do ciclo de criação de 12 meses. Do CTP, $\mathrm{R} \$ 3.418 .500,27(61,37 \%)$ são pertinentes à produção de peixes redondos e $\mathrm{R} \$ 2.151 .532,13(38,63 \%)$ à produção do pintado da Amazônia. Sendo que, dos itens de desembolso a aquisição de ração é o item que apresenta maior participação, em torno de 82,3\%.

Sabaag et al. (2011) trabalharam com a espécie lambari-do-rabo-amarelo em sistema de tanques escavados, também tiveram a ração como principal componente do custo variável, sendo estimada em 62,96\%. Esse valor inferior ao obtido neste trabalho foi devido à ração utilizada para o pintado da Amazônia, que tem hábito alimentar carnívoro, que apresenta valor comercial $49 \%$ maior, quando comparado com ração utilizada para os peixes redondo (hábito alimentar onívoro), devido a maior porcentagem de proteína bruta.

O CV é o que apresenta maior relevância sobre os custos totais com $88,4 \%$ de participação, visto que qualquer variação sobre o mesmo, como por exemplo, a ração, pode influenciar diretamente no CTP. Por conta disso pode se observar a importância de se obter melhor eficiência alimentar na criação, pois o mesmo diminui diretamente o CV (Barros e Martins, 2012).
Outro item de maior importância foi aquisição de juvenis, principalmente do pintado da Amazônia, representando $4,2 \%$ de participação no desembolso (tabela III) devido ao seu elevado valor comercial, quando comparado com outras espécies. Domingues et al. (2014) trabalharam com viabilidade econômica da criação do beijupirá (Rachycentron canadum) e igualmente verificaram a mesma tendência.

O item seguinte que tem representatividade nos custos variáveis são as despesas com mão de obra. Olasunkanmi e Yusuf (2014) enfatizam sobre a importância da eficiência no uso desse recurso, principalmente na criação em pequena escala, pois verificaram que este foi o segundo item mais oneroso do CV, com 31,96\% de participação. Sendo que neste trabalho a participação foi $2,8 \%$ do desembolso. Essa diferença foi explicada por Barros et al. (2010), quando afirmam que, quanto maior é a piscicultura melhor é o emprego da mão de obra, possibilitando melhor utilização da mesma, como também de diversos fatores ligados ao sistema de criação, isso nos mostra que em pisciculturas de pequeno porte existe necessidade de diversificação das atividades.

Os dados relacionados com o custo total de produção estão demonstrados na tabela IV. O valor de 
Tabela IV. Custo total médio de produção e indicadores econômicos de $972.797 \mathrm{~kg}$ de peixes redondo e de $418.639 \mathrm{~kg}$ de pintado da Amazônia em 174 ha de lâmina d'água, Estado de Mato Grosso, Brasil, 2014 (Total production coast and zootechnical indicators of $972.797 \mathrm{~kg}$ of round fish and $418.639 \mathrm{~kg}$ of Amazon pintado on 174 ha of water surface, State of Mato Grosso, Brazil, 2014). (US\$1= R\$2,23: cotação média em Julho de 2014/average price in July 2014).

\begin{tabular}{llccc}
\hline Indicadores econômicos & Valor & Geral & Peixes Redondo & Pintado da Amazônia \\
\hline Custo variável médio & $\mathrm{R} \$ \mathrm{~kg}^{-1}$ & 3,54 & 3,05 & 4,68 \\
Custo fixo médio & $\mathrm{R} \$ \mathrm{~kg}^{-1}$ & 0,47 & 0,47 & 0,46 \\
Custo total médio de produção & $\mathrm{R} \$ \mathrm{~kg}^{-1}$ & 4,00 & 3,51 & 5,14 \\
Preço médio recebido & $\mathrm{R} \$ \mathrm{~kg}^{-1}$ & & 4,00 & 7,00 \\
Receita bruta (RB) & $\mathrm{R} \$$ & $6.821 .660,69$ & $3.891 .189,23$ & $2.930 .471,46$ \\
Margem de contribuição (RB-CV) & $\mathrm{R} \$$ & $1.899 .080,68$ & $925.905,63$ & $973.175,05$ \\
Lucro (RB-CTP) & $\mathrm{R} \$$ & $1.251 .628,29$ & $472.688,95$ & $778.939,33$
\end{tabular}

comercialização praticado na região foi suficiente para cobrir o custo total médio de produção, tanto dos peixes redondo, como do pintado.

Barros e Martins (2012) realizando análises de viabilidade econômica em piscicultura de grande porte obtiveram custo de produção total médio dos peixes redondo de $\mathrm{R} \$ 3,15 \mathrm{~kg}^{-1}$ e preço de comercialização do peixe inteiro foi de $\mathrm{R} \$ 3,21 \mathrm{~kg}^{-1}$, valores inferiores ao encontrado no presente trabalho, o que pode ser explicado pelo seu ano de realização. No entanto, pode se verificar que a situação atual é melhor, uma vez que o preço de venda era $1,9 \%$ mais alto que o CTP e no presente estudo essa diferença passa para 14\% (tabela IV). Isso pode ser decorrente de melhor eficiência no CTP e/ou porque se obteve melhoras em relação ao preço de comercialização.

O custo de produção do pintado é $46,25 \%$ maior que o custo dos peixes redondos, porém seu preço de comercialização é $75 \%$ superior e também apresenta melhores indicadores econômicos (tabela IV). No entanto, a produção não é voltada somente para o pintado da Amazônia por causa da demanda do mercado, que é maior para os peixes redondos e por eles terem mercado estabelecido. E também devido à necessidade de maior capital de giro, uma vez que o preço dos juvenis de pintado e a ração possuem maior valor de mercado.

Os preços de comercialização dos peixes estão sujeitos a mudanças, dependendo do canal de comercialização utilizado, das forças de oferta e demanda pelo produto no mercado bem como a época do ano. Outro fator que vai influenciar diretamente no preço do produto final é o custo de produção, visto que o lucro será maior ou menor dependendo do custo, item que o produtor tem condições de atuar (Nachiluk e Oliveira, 2012), controlando os mesmos para obtenção de maior rentabilidade.

A relação benefício-custo do presente estudo foi de 1,73, valor próximo ao encontrado por Adebayo e Daramola (2013), que realizaram análise econômica da produção do catfish (Clarias gariepinus) na metrópole Ibadan em sistema intensivo e semi-intensivo e encontraram valor da relação benefício-custo de 1,62, demonstrando que a criação de catfish nessa região e em outros sistemas também são rentáveis.

A análise de sensibilidade é uma etapa importante no planejamento e execuções de projetos e tem como objetivo tomar melhores decisões, principalmente com utilização de diferentes indicadores zootécnicos e preços de insumos que refletem nos indicadores econômicos. Na tabela V estão demonstrados os valores de indicadores zootécnicos e financeiros com algumas análises de sensibilidade, como, por exemplo, a ração devido sua maior importância nos custos variáveis, tornou-se interessante à realização de simulações utilizando diferentes CAA.

Uma melhor conversão alimentar aparente acarreta na diminuição da quantidade de ração utilizada e, consequentemente, isso refletirá no lucro ao final do ciclo de criação. No presente trabalho uma economia de $100 \mathrm{~g}$ de ração aumenta o lucro em 17,47\% o que corresponde a $\mathrm{R} \$ 218.644,36$ a mais no final de um ciclo de criação de 12 meses (tabela V).

Quando se aumenta o peso médio final dos peixes redondos de $1,8 \mathrm{~kg}$ para $2,0 \mathrm{~kg}$, se tem pior CAA. Nesse caso a CAA passa a ser de 1,85, elevando em $50 \mathrm{~g}$ e o lucro diminui em 12,93\% (R\$ 61.117,31). No entanto, quando se aumenta o peso do pintado para $2,2 \mathrm{~kg}$ e a CAA em $50 \mathrm{~g}$ também, ocorre redução no lucro de $3,71 \%$ (R\$28.899,01). Essa redução foi menor em função do elevado preço do juvenil, uma vez que se aumenta o peso médio da despesca e não aumenta a biomassa de estocagem, acarretando em menor quantidade de juvenis utilizados. Isso mostra que obter peso final de despesca maior de espécies de peixes cujos juvenis possuem maior valor comercial, mesmo obtendo pior CAA, o impacto negativo no lucro é menor, quando comparado com espécies de juvenis com menor valor de comercialização.

De acordo com as simulações da tabela $\mathbf{V}$, quando se aumenta a BE em 0,100 kg, ou seja, 12,50\%, o lucro aumenta em 24,09\%, isso mostra a importância em se obter maior produtividade. Se ainda for possível aumentar em $25 \%$ a BE $\left(1 \mathrm{~kg} \mathrm{~m}^{-2}\right)$ o lucro passa a ser $49,01 \%$ ( $\$$ \$ 613.443,10) maior, desde que se mantenha a mesma eficiência produtiva, principalmente relacionada à CAA.

No entanto, uma discussão faz-se necessária, pois quando se aumenta a $\mathrm{BE}$ a tendência é que o tamanho final de despesca diminua (Ayrosa et al., 2011; CruzCasallas et al., 2010). Mas, fazendo simulação, com peso final de despesca $10,00 \%$ menor, o lucro passa ser de $22,02 \%$ ( $\mathrm{R} \$ 275.649,20)$, ou seja, com diferença de $2,07 \%$, quando comprado com $24,09 \%$ simulado 
anteriormente. Isso mostra que mesmo que o peso médio final diminua é viável aumentar a biomassa de estocagem.

Se fosse utilizar nesta piscicultura $100 \%$ da área para a criação exclusiva do pintado da Amazônia, seria necessário 31,39\% a mais de capital de giro por ano, porém o lucro seria 91,75\% maior ( $\mathrm{R} \$ 1.197 .896,41)$ quando comprado com a situação inicial do projeto. A viabilidade econômica da criação de bagres (C. gariepinus) também foi verificada por Ugwumba (2011), em sistemas intensivo e semi-intensivo na Nigéria.

No entanto, a criação de peixes redondos é mais significativa, apesar da menor lucratividade, uma vez que existe maior disponibilidade de canais de comercialização e demanda no mercado por esse produto. Este cenário pode ser modificado no futuro, o que dá ao produtor possibilidade de inverter a quantidade de produção das espécies, ou mesmo, acrescentar outras ao sistema de criação.

Outras simulações na análise de sensibilidade (tabela V) apresentaram resultados com maior ou menor viabilidade econômica, porém, nenhuma delas apresentou prejuízo, demonstrando que a piscicultura é uma atividade rentável e que possibilita capacidade de expansão com recurso financeiro próprio, dispensando linhas de crédito oferecidas pelos bancos ou qualquer outro meio para conseguir capital financeiro.

Outro fator que deve ser levado em consideração é a possibilidade de utilização de sistema de policultivo, que tem como objetivo a criação de duas ou mais espécies no mesmo ambiente aquático. A utilização desse sistema na piscicultura do presente estudo seria interessante, pois teria como finalidade aumentar a produtividade da mesma, visto que o policultivo, segundo estudo realizado por Corrêa et al. (2009) aumenta o crescimento e a produção das espécies quando comparadas ao sistema de monocultivo, por causa das interações positivas que acontecem entre as mesmas.

\section{CONCLUSÕES}

A implantação de uma piscicultura no Estado de Mato Grosso, com 174 ha de lâmina d'água em sistemas de tanques escavados com a criação de peixes redondos e pintado da Amazônia, bem como seus índices zootécnicos, apresenta viabilidade econômica, mesmo em diversas situações desfavoráveis como foi apresentado na análise de sensibilidade.

\section{BIBLIOGRAFIA}

Adebayo, O.O. e Daramola, O.A. 2013. Economic analysis of catfish (Clarias gariepinus) production in Ibadan metropolis. Discourse J Agric Food Sci, 1: 128-134.

Ayrosa, L.M.S.; Romagosa, E.; Ayrosa, D.M.M.R.; Scorvo Filho, J.D. e Salles, F.A. 2011. Custos e rentabilidade da produção de juvenis de tilápia-do-nilo em tanques-rede utilizando-se diferentes densidades de estocagem. Rev Bras Zootecn, 40: 231-239.

Barros, A.F.E. and Martins, M.E.G. 2012. Performance and economic indicators of a large scale fish farming in Mato Grosso, Brazil. Rev Bras Zootecn, 41: 1325-1331.

Barros, A.F.; Martins, M.I.E.G.; Abreu, J.S. e Amaral, C.M.C. 2010. Custo total de produção e indicadores econômicos. In: Investimento 
com implantação e custo de produção em pisciculturas no Estado de Mato Grosso. 1. ed. Ed. UNEMAT. Cáceres. 55 pp.

Cruz-Casallas, N.; Marciales-Caro, L.J.; Díaz-Olarte, J.J.; MurilloPacheco, R.; Medina-Robles, V.M. e Cruz-Casallas, P. 2010. Desempeño productivo del yaque (Leiarius marmoratus Gill, 1870) bajo diferentes densidades de siembra en estanques en tierra. Rev Colomb Cienc Pec, 23: 325-335.

Corrêia, V.; Neto, J.R.; Lazzari, R.; Veiverberg, C.A.; Bergamin, G.T.; Pedron, F.A.; Ferreira, C.C.; Emanuelli, T. e Ribeiro, C.P. 2009. Crescimento de jundiá e carpa húngara criados em sistema de recirculação de água. Rev Bras Cien Rural, 39: 1533-1539.

Diário Oficial. 2006. Superintendência da Imprensa Oficial do Estado de Mato Grosso. Decreto-Lei Estadual no 8.464 de 04 de Abril de 2006. Dispõe, define e disciplina a piscicultura no Estado de Mato Grosso e dá outras providencias. Diário Oficial, Cuiabá, n.23.468, p. 1, 04 de abr.

Dieterich, T.G.; Potrich, F.R.; Lorenz, E.K.; Signor, A.A.; Feiden, A. e Boscolo, W.R. 2013. Parâmetros zootécnicos de juvenis de pacu alimentados a diferentes frequências de arraçoamento em tanques-rede. Pesq Agropec Bras, 48: 1043-1048.

Domingues, E.C.; Hamilton, S.; Bezerra, T.R.Q. e Cavalli. R.O. 2014. Viabilidade econômica da criação do beijupirá em mar aberto em Pernambuco. Bol Inst Pesca, 40: 237-249.

Furnaleto, F.P.B. e Esperancini, M.S.T. 2009. Estudo da viabilidade econômica de projetos de implantação de piscicultura em viveiros escavados. Inform Econ, 39: 5-11.

Gonçalves, A.C.S.; Murgas, L.D.S.; Rosa, P.V.; Navarro, R.D.; Costa, D.V. e Teixeira, E.A. 2010. Desempenho produtivo de tambacus alimentados com dietas suplementadas com vitamina E. Pesq Agropec Bras, 45: 1005-1011.

Izel, A.C.U. e Melo, L.A.S. 2004. Criação de tambaqui (Colossoma macropomum) em tanques escavados no Estado do Amazonas. Embrapa Ocidental. Brasília. Documento 32. Agosto. 14 pp.

Martins, M.I.E.G. e Borba, M.M.Z. 2008. Custo de produção. FCAV/ UNESP. Jaboticabal. $22 \mathrm{pp}$
Martin, N.B.; Scorvo Filho, J.D.; Sanches, E.D.; Novato, P.F.C. e Ayrosa, L.M.S. 1995. Custo e retornos na piscicultura em São Paulo. Inform Econ, 25: 9-47.

Melo, L.A.S. Izel, A.C.U. e Rodrigues, F.M. 2001. Criação de tambaqui (Colossoma macropomum) em viveiros de Argila/Barragens no Estado do Amazonas. Embrapa Ocidental. Manaus. Documento 18. Dezembro. 36 pp.

Nachiluk, K. e Oliveira, M.D.M. 2012. Custo de produção: uma importante ferramenta gerencial na agropecuária. Análises e Indicadores do Agronegócio, 7: 1-7.

Olasunkanml, N.O. e Yusuf, O. 2014. Resource use efficiency in small scale catfish farming in Osun State. Nigeria. Sky J Agric Res, 3: 037-045.

Oliveira, A.M.S; Oliveira, C.A.L.; Rodrigues, R.A.; Sanchez, M.S.S.; Nunes, A.L.; Fantini, L.E. e Campos, C.M. 2014. Crescimento de juvenis de Pseudoplatystoma reticulatum e Pseudoplatystoma spp. em viveiro. Semin Cienc Agrar, 35: 1091-1098.

Sabaag, O.J.; Rozales, R.R.; Tarsitano, M.P.A. e Silveira, A.N. 2007. Análise econômica da produção de tilápias (Oreochromis niloticus) em um modelo de propriedade associativista em Ilha Solteira/SP. Custos e @gronegócio on line, 3: 86-100.

Sabbag, O.J.; Takahashi, L.S.; Silveira, A.N. eAranha, A.S. 2011 . Custos e viabilidade econômica da produção de lambari-do-rabo-amarelo em Monte Castelo-SP: Um estudo de caso. Bol Inst Pesca, 37: 307-315.

Scorvo Filho, J.D.; Martins, M.I.G.E. e Frasca-Scorvo, C.M.D. 2004. Instrumento para análise da competitividade na piscicultura. In: Crino, J.E.P.; Urbinati, E.C.; Fracalossi, D.M. e Castagnolli, N. (Eds.). Tópicos especiais em piscicultura de água doce tropical intensivo. Tec Art. São Paulo. pp. 517-533.

Ugwumba, C.O.A. 2011. Analysis of catfish farming system and its impacto net farm income in Anambra State. Nigeria. ARPN J Agric Biol Sci, 6: 26-30.

Vilela, M.C.; Araújo, K.D.; Machado, L.S. e Machado, M.R.R. 2013. Análise da viabilidade econômico-financeira de projeto de piscicultura em tanques escavados. Custos e @gronegócio on line, 9: 154-173. 